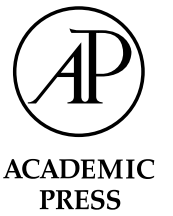

\title{
Ultrasensitive investigations of biological systems by fluorescence correlation spectroscopy
}

\author{
Elke Haustein and Petra Schwille* \\ Experimentelle Biophysik, Max-Planck-Institut für biophysikalische Chemie, Am Fassberg 11, D-37077 Göttingen, Germany
}

Accepted 15 October 2002

\begin{abstract}
Fluorescence correlation spectroscopy (FCS) extracts information about molecular dynamics from the tiny fluctuations that can be observed in the emission of small ensembles of fluorescent molecules in thermodynamic equilibrium. Employing a confocal setup in conjunction with highly dilute samples, the average number of fluorescent particles simultaneously within the measurement volume $(\sim 1 \mathrm{fl})$ is minimized. Among the multitude of chemical and physical parameters accessible by FCS are local concentrations, mobility coefficients, rate constants for association and dissociation processes, and even enzyme kinetics. As any reaction causing an alteration of the primary measurement parameters such as fluorescence brightness or mobility can be monitored, the application of this noninvasive method to unravel processes in living cells is straightforward. Due to the high spatial resolution of less than $0.5 \mu \mathrm{m}$, selective measurements in cellular compartments, e.g., to probe receptor-ligand interactions on cell membranes, are feasible. Moreover, the observation of local molecular dynamics provides access to environmental parameters such as local oxygen concentrations, $\mathrm{pH}$, or viscosity. Thus, this versatile technique is of particular attractiveness for researchers striving for quantitative assessment of interactions and dynamics of small molecular quantities in biologically relevant systems.
\end{abstract}

(c) 2003 Elsevier Science (USA). All rights reserved.

Keywords: Fluctuations; FCS; Diffusion; Membrane

\section{Introduction}

Thirty years ago, Webb and Coworkers [1-3] published a rigorous formalism of fluorescence correlation spectroscopy (FCS) with its various modes of possible applications, highlighting the large potential of this new variant of relaxation methods. In general, relaxation methods subject a reaction system to sudden, extreme changes of the thermodynamic state variables, taking it far from thermal equilibrium and facilitating study of the subsequent relaxation. In contrast to classical techniques that require quite substantial alterations of environmental conditions, FCS and related fluctuation relaxation analyses utilize small fluctuations around thermal equilibrium inherent in any system at finite temperatures to extract the same kind of dynamic information. The final breakthrough of this novel method, however, did not occur until Rigler et al. [4,5] actually

\footnotetext{
${ }^{*}$ Corresponding author. Fax: +49-551-201-1435.

E-mail address: pschwil@gwdg.de (P. Schwille).
}

reached the single-molecule detection limit by combining FCS with a confocal setup, thus increasing the signal-to-noise ratio dramatically. By tightly focusing a laser beam and inserting a pinhole into the image plane, maximum lateral and axial resolution can be achieved. With the sample volume under investigation being now only of about the size of an Escherichia coli bacterial cell, the collection of unwanted background light can be suppressed far below the fluorescence signal obtainable from a single fluorogenic molecule. Because the limitation of the small measurement volume is purely optical and no mechanical constraints are induced, this comparatively noninvasive technique is ideally suited for both in vitro and in vivo measurements, the latter benefiting especially from the spatial selectivity due to the small illuminated region.

Any molecular process causing an alteration in the emission characteristics of the fluorophore can be monitored with high precision. The resolvable time scales are limited only by the temporal resolution of the measurement electronics on the one side and by the 
dwell times of the fluorescent particles within the focal volume on the other side. The first parameters that could be determined with high precision utilizing the new, single-molecule-based application mode of FCS were concentrations, diffusion constants in aqueous environments [5], and association rates of slow intramolecular reactions altering the molecular mobility [6-9]. Among the possible environmental parameters that are accessible by observing internal fluorescence fluctuations of single dye molecules are local $\mathrm{pH}$, viscosity, and concentration of molecular oxygen [10].

In 1994, Eigen and Rigler [11] triggered an important further development by proposing the application of dual-color cross-correlation (FCCS) for diagnostic purposes. The underlying idea was to separate singlelabeled reaction educts from dual-labeled reaction products to discriminate against excess of free singlelabeled species and thus enhance specificity of detection. The experimental realization of FCCS followed in 1997, when Schwille et al. [12] successfully monitored a hybridization reaction of two differently labeled oligonucleotides. Although the proposed advantages of FCCS for diagnostic purposes could not be verified before 2000 when Bieschke et al. [13] showed its impact to study early aggregation steps of pathogenic prion protein, the impact of the cross-correlation mode to significantly simplify studies of nucleic acid and/or protein interaction turned out to be substantial [14]. Its most striking in vitro application to date was certainly the employment in quantitative analysis of enzymatic reactions, as first demonstrated by Kettling et al. [15]. A crucial new technical improvement with particular relevance for future cellular FCCS applications occurred in 2000, when Heinze et al. [16] reported the application of twophoton cross-correlation to determine enzymatic cleavage of a DNA substrate by EcoRI endonuclease.

Meanwhile, FCS has evolved into a whole family of related methods sharing the basic principle of fluorescence fluctuation analysis, yet displaying subtle differences in applicability and experimental realizations. Therefore, after a brief introduction to the theoretical and experimental background, a variety of applications will be discussed.

\section{Methods}

\subsection{FCS Theory}

The normalized fluorescence fluctuation autocorrelation function $G_{i i}(\tau)$ with lag time $\tau$ is defined as

$G_{i i}(\tau)=\left\langle\delta F_{i}(t) \delta F_{i}(t+\tau)\right\rangle /\left\langle F_{i}(t)\right\rangle^{2}$

The fluorescence fluctuations $\delta F(t) \equiv F(t)-\langle F(t)\rangle$ induced by an arbitrary process occurring on the molecular level depend on various physical parameters, e.g., the overall fluorescence detection efficiency and the spatial distribution of the excitation intensity.

Provided that the fluctuations are due only to changes in concentration (so-called number fluctuations), this leads to the following expression for the normalized three-dimensional (3D) diffusion autocorrelation function for species $i$ :

$G_{i i}(\tau)=V_{\text {eff }}^{-1}\left\langle C_{i}\right\rangle^{-1}\left(1+\tau / \tau_{\mathrm{d}, i}\right)^{-1}\left(1+r_{0}^{2} \tau / z_{0}^{2} \tau_{\mathrm{d}, i}\right)^{-1 / 2}$,

where $\tau_{\mathrm{d}, i}=r_{0}^{2} / 4 D_{i}$ is defined as the average lateral diffusion time for a molecule of species $i$ through the effective measurement volume element $V_{\text {eff }}=\pi^{3 / 2} r_{0}^{2} z_{0}$ [12]. Thus, the diffusion coefficient can be easily derived from the characteristic decay time of the correlation function $\tau_{\mathrm{d}, i}$ in calibrated systems with known $V_{\text {eff }}$. Using the relationship $\left\langle C_{i}\right\rangle=G_{i i}(0)^{-1} V_{\text {eff }}^{-1}$, the local concentration of fluorescent molecules can also be determined from the measured correlation curve amplitude $G(0)$ with very high precision. For approximately spherical particles, the local viscosity $\eta_{\mathrm{V}}$ of the medium can be obtained from the diffusion coefficient via the Stokes-Einstein equation

$D_{i}=\frac{k T}{6 \pi \eta_{\mathrm{V}} R_{h, i}}$.

The parameter $T$ is the temperature, $k$ is Boltzmann's constant, and $R_{\mathrm{h}, i}$ is the hydrodynamic radius of the particles. If $R_{\mathrm{h}, i}$ is unknown, comparative studies with diffusing molecules in another environment are required.

If particle motion is governed not only by translational $3 \mathrm{D}$ diffusion or if several particle species of different mobility and/or emission characteristics contribute to the fluorescence signal, the expression in Eq. (7) is generalized as

$G_{\mathrm{M}}(\tau)=V_{\text {eff }}^{-1} \sum_{n} \eta_{n}\left\langle C_{n}\right\rangle M_{n}(\tau) /\left(\sum_{n} \eta_{n}\left\langle C_{n}\right\rangle\right)^{2}$

with

(a) $M_{n}(\tau)=\left(1+\tau / \tau_{\mathrm{d}, n}\right)^{-1}\left(1+r_{0}^{2} \tau / z_{0}^{2} \tau_{\mathrm{d}, n}\right)^{-1 / 2}$

for free 3D diffusion,

(b) $M_{n}(\tau)=\left(1+\tau / \tau_{\mathrm{d}, n}\right)^{-1}$

for free 2D membrane diffusion,

(c) $M_{n}(\tau)=\exp \left(-\left(\tau \cdot v / r_{0}\right)^{2}\right)$

for active transport with velocity $v$.

In complex biological environments, the phenomenon of anomalous (restricted) subdiffusion has to be mentioned [17]. Although a multitude of factors can induce deviations from normal (Brownian) diffusion, each of them strictly requiring rigorous mathematical modeling, anomalous diffusion can often be generically accounted for by assuming that the mean square displacement is nonlinear with time, $\left\langle r^{2}\right\rangle \sim t^{\alpha}$ with $\alpha<1$, and by replacing the expression $\tau / \tau_{\mathrm{d}, n}$ by $\left(\tau / \tau_{a n, n}\right)^{\alpha}[17,18]$. In this case, no conventional diffusion constant can be speci- 
fied. Instead, the transport coefficient $\Gamma_{n}$ of fractional time dimension is given by $\tau_{a n, n}^{\alpha}=r_{0}^{2} / \Gamma_{n}$. Model curves showing the differences between the four above-mentioned mobility modes can be seen in Fig. 1. The autocorrelation curve for active transport displays the sharpest decay, whereas the one for anomalous diffusion decreases rather slowly. If one treats the autocorrelation curve as a kind of distribution function of residence times within the focal volume, this behavior becomes clearer. Particles diffusing within a biological membrane may exhibit a large variety of different mobilities, depending on the time-dependent local environment of each individual chromophore. This results in curves that are typically smeared out across several orders of magnitude in time. The exact opposite situation is physically realized by plug flow experiments. Here, all particles are expected to have the same velocity. Only the ellipsoidal shape of the focal volume causes a small variation in the residence times, so that the resulting decay of the autocorrelation curve is merely very abrupt, but not strictly perpendicular. The same principles may be applied to two- and three-dimensional diffusion, respectively. Because of the cigar-shaped focus, the times spent within this region differ to a much greater extent if the particle is free to diffuse in space and is not restricted to a planar motion.

Hitherto, during a molecule's presence in the illuminated volume a constant fluorescence capacity, $\delta \eta_{i}=0$, was assumed. In reality, however, the dynamic fluorescence properties for most dye systems are much more complex and may be influenced by the molecular environment. The most prevalent phenomenon is induced by the reversible transition of the dye to the quantum-mechanically forbidden, the long-living first excited triplet state $[10,19]$. The resulting repetitive fast fluorescence intermittence can be seen in the correlation curves as additional shoulders in the fast time range. Hence, the mobility-dependent correlation curves $G_{\mathrm{M}}(\tau)$ from Eq. (4) have to be supplemented by an exponential decay term,

$G_{\mathrm{M}, \mathrm{T}}(\tau)=G_{\mathrm{M}}(\tau)\left(1-T+T \mathrm{e}^{-t / \tau_{\mathrm{T}}}\right)$

In Fig. 2, a model autocorrelation curve is depicted and the individual parameters governing special features are pointed out. Eq. (5) can be generalized for any interand intramolecular reaction inducing fluctuations on much faster time scales than the mobility-related number fluctuations. If the diffusion coefficients of the molecules remain unaltered, the overall correlation curve is given by the product of a reaction term $X(\tau)$ with the mobility term $G_{\mathrm{M}}(\tau)[20]$ :

$G_{\mathrm{tot}}(\tau)=X(\tau) G_{\mathrm{M}}(\tau)$.

Considering reversible transitions between the fluorescent state B and a fully dark state D,

$\mathrm{B} \underset{k_{\mathrm{B}}}{\stackrel{k_{\mathrm{D}}}{\rightarrow}} \mathrm{D}$

the same functional form as that for the triplet dynamics in Eq. (5) applies. Thus, $X(\tau)=\left(1-F+F \mathrm{e}^{-t / \tau_{F}}\right)$ with the relaxation time $\tau_{F}=\left(k_{\mathrm{D}}+k_{\mathrm{B}}\right)^{-1}$ and $F=k_{\mathrm{D}} /$ $\left(k_{\mathrm{D}}+k_{\mathrm{B}}\right)$ as the average fraction of dark molecules or, respectively, the average fraction of time that a molecule spends in the dark state.

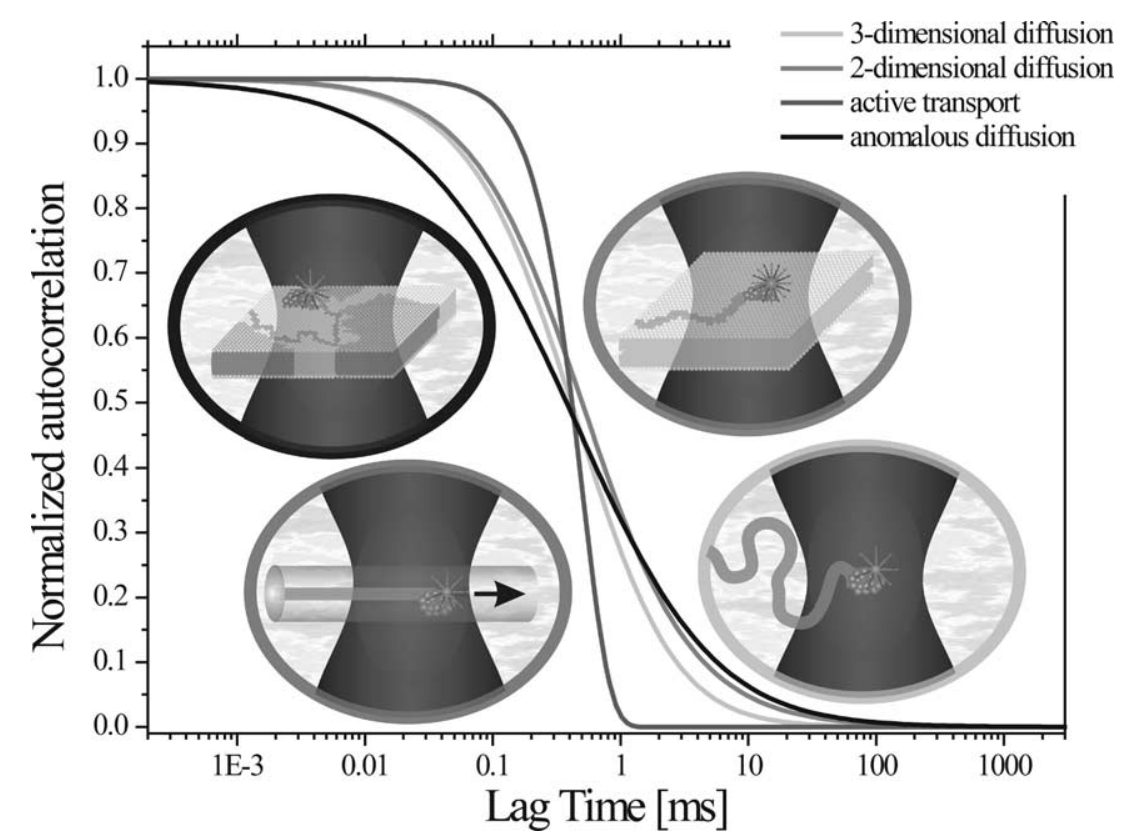

Fig. 1. Model autocorrelation curves for different particle mobilities: free three- and two-dimensional diffusion, active transport, and anomalous diffusion. See text for additional information. 


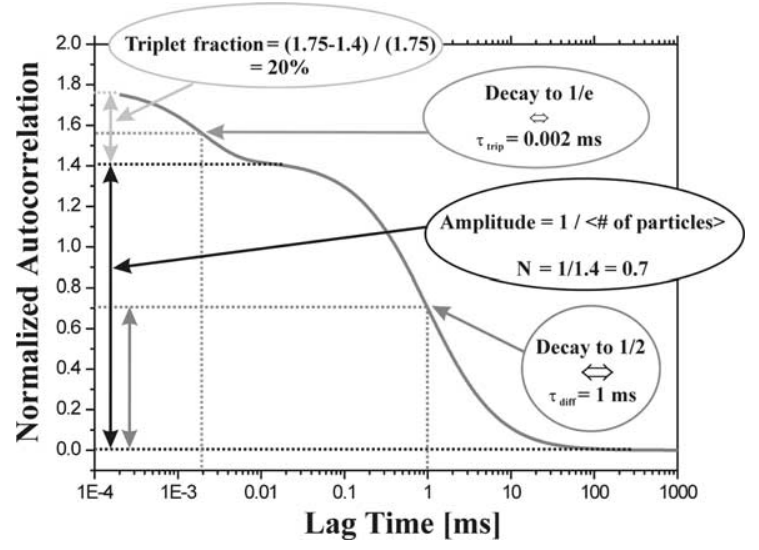

Fig. 2. Various parameters can be extracted from this model autocorrelation curve displaying two dynamic processes on clearly separated time scales. The slower process can be attributed to the diffusion of the particles in and out of the focal volume. The inverse amplitude of this process equals the average particle number within the illuminated region and the half-value decay time can be attributed to the mean diffusion time. The same principle can be applied to the faster triplet blinking. The fraction of molecules undergoing transitions to the triplet state can also be calculated via the amplitude, whereas the "blinking time" is given by the decay of this part of the curve to $1 / e$.

Cross-correlation. Performing an autocorrelation analysis, one effectively compares a measured signal with itself at some later time and looks for recurring patterns. Nevertheless, it may also be useful to find common features in two independently measured signals. In fact, cross-correlation analysis is just the straightforward way to generalize the method described above and thus achieve much higher detection specificity. In analogy to Eq. (1), the cross-correlation function is defined as

$G_{i j}(\tau)=\left\langle\delta F_{i}(t) \delta F_{j}(t+\tau)\right\rangle /\left\{\left\langle F_{i}(t)\right\rangle\left\langle F_{j}(t)\right\rangle\right\}$.

The most prominent example of cross-correlation is the dual-color mode schematically depicted in Fig. 3. Whereas autocorrelation measurements are sensitive only to signal variations within one channel, cross-correlation analysis is used to compare the signals arising from two differently labeled fluorescent species. Only temporally coordinated fluctuations in both channels, i.e., colors, give rise to cross-correlation. Phenomena limited to one fluorophore, e.g., triplet blinking, or artifacts in one detector will not show in the cross-correlation curve, because they are not directly related to the other channel. The two spectrally different dyes are excited within the same detection element using two overlapping laser beams and separate detection pathways $[12,14,21]$. Dual-color cross-correlation is an extremely powerful tool to probe interactions between different molecular species, and a number of experiments applying this technique to different kinds of reactions have been carried out. The basic measurement scheme is quite simple: the two differently labeled educts move independently at first and are then fused together

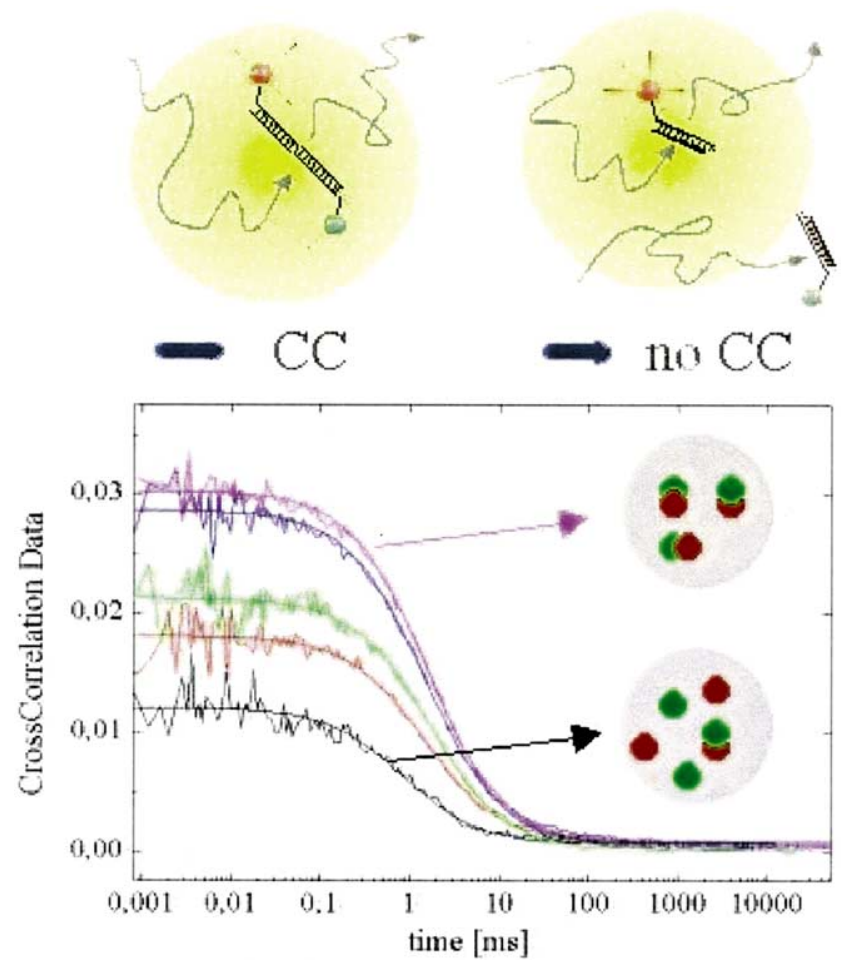

Fig. 3. The amplitude of the autocorrelation curve is a direct measure of the number of double-labeled particles. Only coordinated movements of the two different chromophores result in non-zero crosscorrelation amplitudes. For an enzymatic cleavage reaction of a doubly labeled DNA strand, the maximum amplitude will be measured before starting the reaction. The cleavage reaction can be monitored online via the gradually decreasing cross-correlation amplitude that directly reflects the decreasing number of double-labeled particles. In the theoretical limit of all DNA having been processed, the amplitude should have dropped to zero in an ideal measurement.

during the reaction or vice versa. The motion of the different components is supposed to be described each by the term $M_{i}(\tau)$ (cf. Eq. (4)). Assuming ideal conditions, where both channels have the same effective volume element $V_{\text {eff }}$, fully separable emission spectra, and a negligible emission-absorption overlap integral, the following correlation curves can be derived:

Autocorrelation

$G_{i}(\tau)=\frac{\left(\left\langle C_{i}\right\rangle M_{i}(\tau)+\left\langle C_{12}\right\rangle M_{12}(\tau)\right)}{V_{\text {eff }}\left(\left\langle C_{i}\right\rangle+\left\langle C_{12}\right\rangle\right)^{2}}$

with $i=1,2$

Cross-correlation

$G_{x}(\tau)=\frac{\left\langle C_{12}\right\rangle M_{12}(\tau)}{V_{\text {eff }}\left(\left\langle C_{1}\right\rangle+\left\langle C_{12}\right\rangle\right)\left(\left\langle C_{2}\right\rangle+\left\langle C_{12}\right\rangle\right)}$

$C_{i}(\underline{r}, t)$ is the concentration of the single-labeled species $i(i=1,2)$ and $C_{12}(\underline{r}, t)$ that of the double-labeled species [22] respectively. If there is no reaction-induced quenching or fluorescence enhancement and no particle 
exchange in the sample, the amplitude of the crosscorrelation function is at any time directly proportional to the relative fraction of double-labeled particles in the volume element. Knowing the amplitudes of the autocorrelation curves and thus the concentrations of both single-labeled species, the absolute concentration $\left\langle C_{12}\right\rangle$ can be determined from Eq. (8) as follows:

$$
\left\langle C_{12}\right\rangle=\frac{G_{x}(0)}{G_{1}(0) G_{2}(0) V_{\text {eff }}} .
$$

Two-photon correlation and cross-correlation. One of the major drawbacks of the confocal setup described above consists in the fact that axial resolution can be achieved only for detection, by inserting a pinhole into the image plane. Although this approach efficiently suppresses the amount of background light and restricts the system under observation, it does not prevent molecules outside the focal region from being excited and eventually photobleached.

Denk et al. [23,24] were the first to demonstrate that two-photon excitation provides intrinsic $3 \mathrm{D}$ resolution in laser scanning microscopy, thereby confining photodamage to the immediate vicinity of the focus (cf. Fig. 4). Hence, selective illumination of interesting sites in the living cell becomes practicable. By employing twophoton excitation for intracellular FCS [25], the same
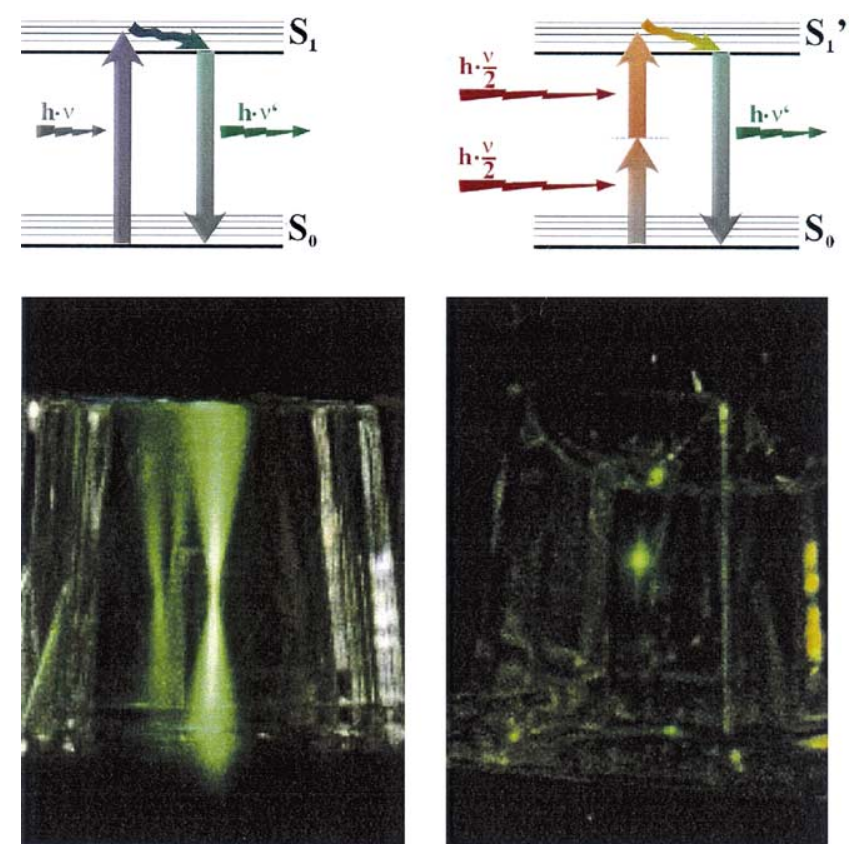

Fig. 4. Photograph of the laser focus for one-photon (left) and twophoton (right) excitation. In the first case, a double cone is illuminated and spatial resolution has to be enforced by inserting a pinhole into the image plane. The latter, however, displays inherent sectioning. Only molecules within a region providing sufficiently high intensities - and thus nonzero probability for the quasisimultaneous absorption of two photons - are excited. The different underlying absorption processes can be seen at the top. advantages as those previously reported for imaging applications could be experimentally verified. In comparison to conventional one-photon FCS, two-photon excitation at the same signal levels minimizes photobleaching in spatially restrictive cellular compartments, thereby preserving long-term signal acquisition. Nevertheless, it has to be pointed out that two-photon-induced transitions to the excited state are formally symmetry forbidden and thus exhibit different selection rules and vibronic coupling. As a consequence, the twophoton excitation spectra of many common fluorophores differ considerably from the respective (doubled) one-photon spectra while no change in emission properties can be observed. Although this renders determination of the optimal two-photon excitation conditions rather tedious, simultaneous excitation of spectrally different dyes becomes feasible. Experimental implementations comprise confocal imaging applications [26] and, more recently, single-molecule-based techniques. The latter require the detection of two labels on a single molecule within the limited time frame of a molecule's dwelling in the focal spot. Even the intrinsic fluorescence of tryptophan-containing proteins has recently been used for recording autocorrelation curves via two-photon excitation [27].

The experimental setup for two-photon cross-correlation spectroscopy is significantly simplified compared to confocal cross-correlation geometries, because only one laser line is used for excitation and no pinholes are in principle required in the detection pathway. Because of the quadratic dependence of fluorescence on excitation intensity, both the effective volume element and consequently the diffusion times change. Here, $\tau_{d, i}=r_{0}^{2} / 8 D_{i}$ is the average lateral diffusion time for a molecule of species $i$ through the effective measurement volume element $V_{\text {eff }, 2 P E}=(\pi / 2)^{3 / 2} r_{0}^{2} z_{0}$ [16]. Apart from that, for ideally separated channels the same formalism as that for one-photon cross-correlation measurements described above applies.

\section{Instrumentation}

A typical FCS setup basically consists of an inverted microscope rig (e.g., Olympus IX70) in combination with a high-numerical aperture objective (cf. Fig. 5). Preferably, the numerical aperture should be larger than 0.9 . For excitation, a variety of single-mode lasers are suitable, such as argon ion lasers, frequency-doubled NdYAG lasers, or helium-neon single-line tubes. Laser diodes provide an inexpensive alternative. For twophoton (and pulsed) excitation, a tunable titaniumsapphire laser operating at $100 \mathrm{fs} / 80 \mathrm{MHz}$ pulse width and repetition rate may be the best choice.

The incoming parallel laser light is reflected by the dichroic mirror and focused by the microscope objective 


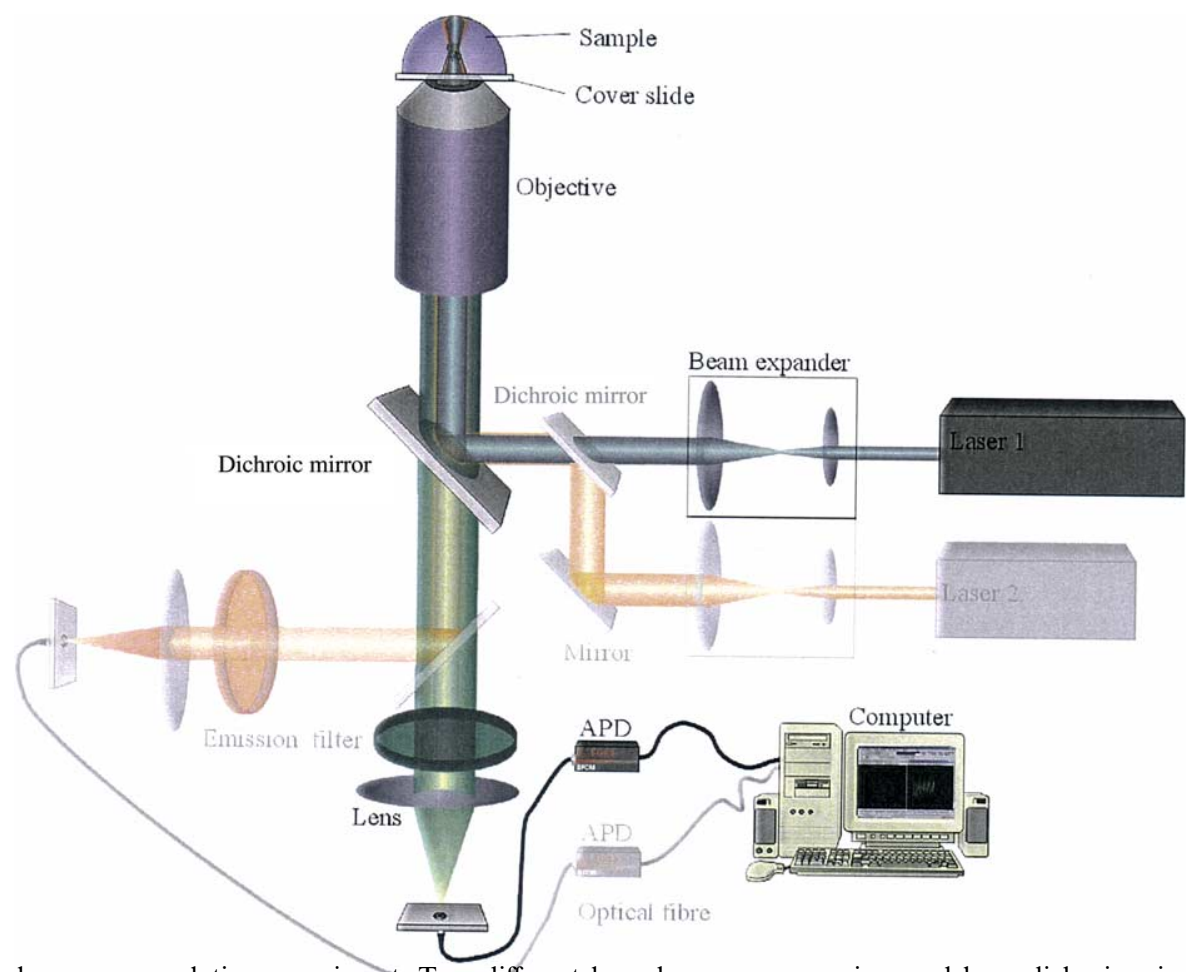

Fig. 5. Setup for a dual-color cross-correlation experiment. Twe different laser beams are superimposed by a dichroic mirror and focused by a microscope objective into the sample. Fluorescence light is collected by the same objective. The two different colors are separated by another dichroic mirror. Residual excitation light is removed by bandpass filters before the light is directed onto the detectors. The fluorescence signals are hardwarecorrelated by a commercial PC card. If the elements drawn in lighter colors are omitted, one gets the standard autocorrelation setup.

to a spot of approximately $0.5 \mu \mathrm{m}$ diameter. Filling the back aperture to approximately $2 / 3$ is usually an ideal compromise for minimizing both the size of the confocal volume and the optical aberrations. The red-shifted fluorescence light passes the dichroic and the emission filter that removes residual Rayleigh and Raman scattered light. In Fig. 6, the effect of the different filters on the transmitted and reflected light of the two fluorophores can be seen.

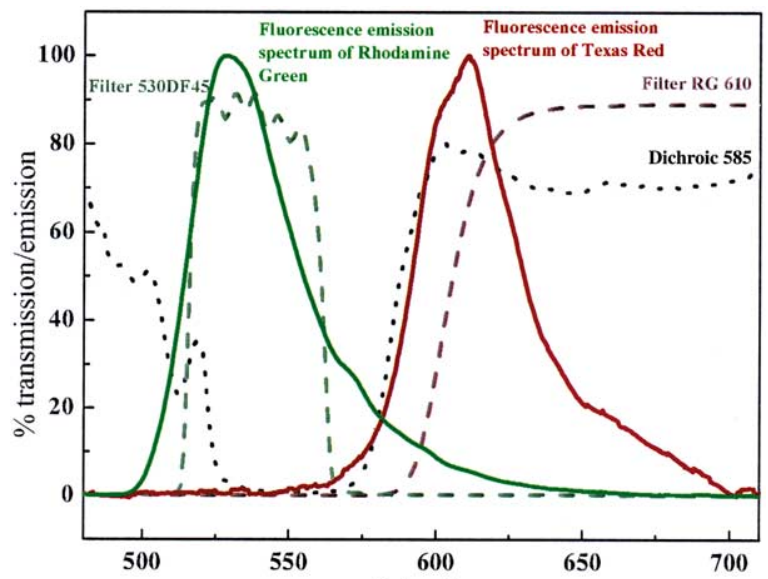

Fig. 6. Fluorescence emission spectrâ bntwwo fluorescent dyes (rhodamine green and texas red) and the appropriate filter system for a crosscorrelation setup. Courtesy of Katrin Heinze.
For one-photon excitation, inserting a pinhole into the image plane is enforcing axial resolution. Alternatively, optical fibers of the same core diameter can be used (multimode fibers; Ocean Optics). Due to the inherent sectioning of two-photon excitation, the pinhole may be omitted.

The most commonly used detectors are avalanche photodiodes with single-photon sensitivity (EG\&G). Especially in the blue spectral region, however, photomultipliers might also be considered. Depending on the application, hardware correlators such as the ALV-5000E multiple- $\tau$ correlator card (ALV, Langen, Germany) or an USB-based hardware correlator from correlator.com will give satisfying results. Nevertheless, it is also possible to record the fluorescence time trace and correlate the data afterward. Unless a commercial setup with built-in fitting algorithms is used, data evaluation can be performed using appropriate model functions and Levenberg-Marquardt nonlinear leastsquares fitting routines provided by any good math program [28].

Especially for those users who are primarily interested in potential biological applications and do not want to bother with the nuts and bolts of a complicated custom-made setup, commercial systems (e.g., ConfoCorII; Zeiss, Jena) are meanwhile available either as stand-alone versions or combined with a laser scanning microscope. 


\section{Applications}

\subsection{Concentration and aggregation measurements}

Now, what actually can be learned from the autocorrelation amplitudes about a particular system? To find an answer, one must look more closely at Eq. (2). It is evident that $G(0)$ equals the reciprocal number of molecules $N$ in the effective volume element $V_{\text {eff }}$. Thus in principle, absolute local concentrations can be determined very precisely if the size of the confocal volume element $V_{\text {eff }}$ is known $[9,11,29]$. For some studies, however, relative numbers are sufficient. Kinjo and $\mathrm{Na}-$ shimura [30], for example, followed the cleavage of fluorescently labeled DNA with three kinds of restriction enzymes (HaeIII, HgaI, and BsmAI) by monitoring the number of DNA fragments using FCS. Based on these studies, they suggest FCS as a possible tool for finding genetic differences in DNA sequences.

Nevertheless, it is advisable to take utmost care when trying to figure out chemical kinetics by monitoring increasing or decreasing particle numbers. A large number of control measurements is recommended before confidence in these results can be achieved, because most dilute substances are prone to surface adsorption. Coating the reaction chambers, e.g., with bovine serum albumin, may be one possible solution to this dilemma. In any case, because of the extremely high sensitivity of this method, one needs to make absolutely sure that no traces of other fluorescent substances are present in the solution. Therefore testing of all buffers, vessels, etc. beforehand is advised.

Even if everything else works perfectly, there may still be severe difficulties when monitoring association or dissociation reactions in which the fluorophore is severely quenched in one of the two possible states. Not only may dramatic differences in brightness cause artifacts in the recorded curves, but they also need elaborate theoretical treatment.

\subsection{Mobility analysis}

The determination of mobility-related parameters of biologically relevant molecules is one of the primary goals of FCS analysis in aqueous solution and one that it is especially suited for. The submicrometer spatial resolution also makes it a useful technique for intracellular measurements. When trying to figure out how biological processes work in detail, it is essential to distinguish between diffusion or active transport, anomalous subdiffusion, or even convection. Signal transduction or metabolic regulatory pathways can be understood only once the underlying transport mechanisms are revealed and well characterized. Because pioneers of the FCS technique initially had to struggle with background suppression and unwanted photobleaching, the fluorescence recovery after photobleaching (FRAP) method has long been preferred for in vivo motility studies. The latter requires much higher dye concentrations and is thus less liable to suffer from the autofluorescence background (for detailed discussion of FCS versus FPR, see [31]). However, FRAP often requires unreasonably high fluorophore concentrations and its temporal resolution is mainly limited to the millisecond time scale, such that FCS offers both higher dynamic performance and increased sensitivity. Nanomolar chromophore concentrations and low laser power suffice for this equilibrium measurement, which is definitely less stressful for the cells under investigation. However, because of this inherent sensitivity, the proper selection of dyes for FCS is crucial. One must always be aware of the fact that the dyes might interfere with the monitored mechanism or introduce their own dynamics to the system. Many standard dyes, such as rhodamines and cyanines, are highly lipophilic and tend to associate to intracellular membranes, inducing severe deviations from free diffusion in the cytosol [32].

In Fig. 7, a selection of measurements is depicted. It is quite obvious that the mobility of the fluorophores strongly depends on the environment. The half-value decay time of the curves, which allows for a crude estimation of the mobility of the chromophore, varies by several orders of magnitude between the small dye in the buffer and the large labeled receptor on the plasma membrane. The corresponding diffusion coefficients that can be calculated from the decay times using Eq. (4) range from $3 \times 10^{-6} \mathrm{~cm}^{2} / \mathrm{s}$ for the free dye to $10^{-10} \mathrm{~cm}^{2} /$ $\mathrm{s}$ for the bulky receptor.

\subsection{Confined and anomalous diffusion}

It is known from other techniques such as FRAP or particle tracking [33] that membrane-bound receptors often exhibit anomalous subdiffusion. As outlined above, the mean square displacement of the molecules is in this case not proportional to the observation time $t$, but exhibits fractional time dependence [34,35]. Among possible reasons for such a non-Brownian behavior are environmental heterogeneities (e.g., different lipid phases or rafts), nonspecific interactions with other particles, or local confinement. The underlying principles for nonhomogeneous diffusion can be only speculated. It is quite astonishing that not only membrane-bound proteins exhibit such a strange motility, but also the lipids themselves show deviations from the normal homogeneous diffusion in natural cell membranes [17]. To exclude any label-induced artifacts, measurements in single-phase model membranes of giant unilamellar vesicles have been performed. In these idealized model systems, the lipids indeed show a perfectly homogeneous 2D Brownian diffusion. An altered membrane composition of the model membranes, resulting in a distinctive 

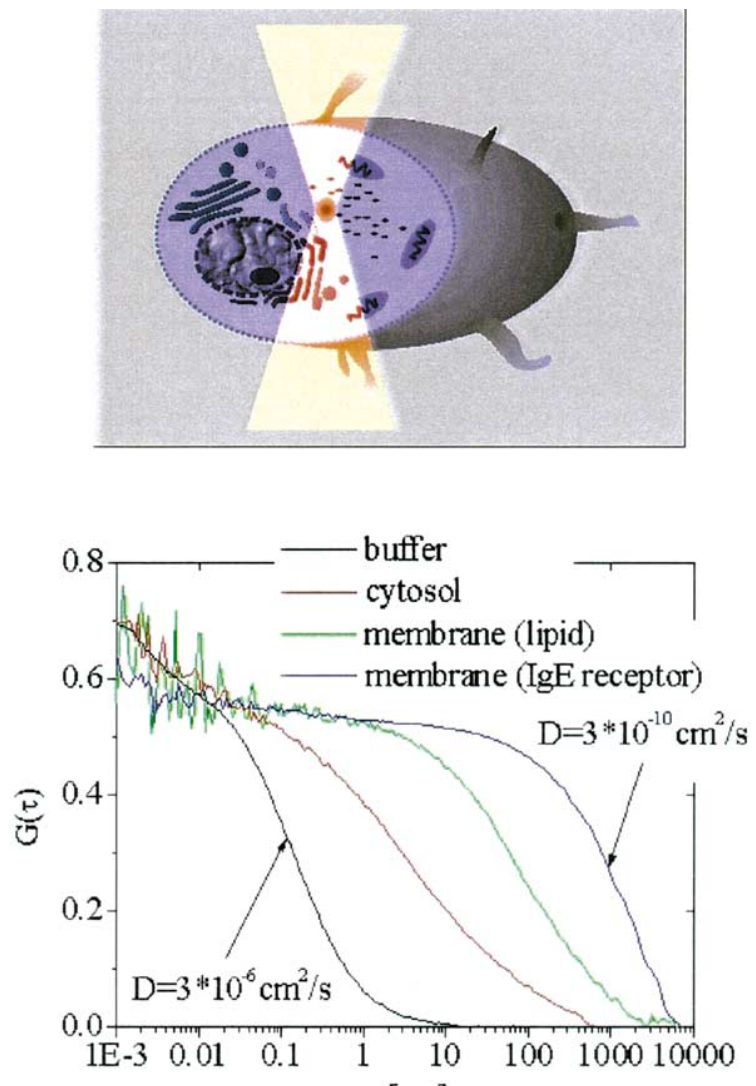

Fig. 7. Autocorrelation curves in different molecular environments. Depending on the surrounding medium, the molecular mobility changes by several orders of magnitude. In aqueous buffer solution, the diffusion coefficient was about $3 \times 10^{-6} \mathrm{~cm}^{2} / \mathrm{s}$. For the large receptor in the cell membrane, mobility was severely decreased. Here, the determined diffusion coefficient was approximately four orders of magnitude lower.

phase separation, however, led to multiple diffusion coefficients for the labeled lipids [36]. Therefore one might assume that environmental heterogeneities play a key role in the anomalous subdiffusion phenomenon.

\subsection{Active transport phenomena}

In 1978, the theoretical background for analyzing different active transport processes such as laminar flow or a combination of directed plug flow and diffusion was laid [37]. Whereas there are examples describing FCS analysis of a laminar flow obeying Hagen-Poiseuille's law or the uniform electroosmotic flow (P. Dittrich, unpublished), measurements of active transport phenomena in living cells are still scarce and will be discussed in more detail at the end of this review. From Figs. 1 and 7 , it is nevertheless evident that FCS is ideally suited to distinguish between different forms of mobilities.

\subsection{Conformational changes}

But FCS is not only applicable to molecular motions, there are also various ways in which conformational changes can be assessed. The diffusion coefficient of a particle in an aqueous solution of viscosity $\eta_{\mathrm{V}}$ is given by the Stokes-Einstein relationship (Eq. (3)). Changes in the diffusion coefficient can be induced not only by chemical reactions but also by any other phenomenon causing changes in the hydrodynamic radius $R_{\mathrm{h}}$. Therefore any variations in the hydrodynamic radius will directly affect the mobility of the molecule. A more massive particle will usually have a larger volume and thus an increased $R_{\mathrm{h}}$. However, the three-dimensional structure and, thus, the shape of a protein also influence its mobility, such that it is in principle possible to monitor protein folding or unfolding transitions. The differences in the diffusion coefficients in two folding states are generally quite small; this is why only few measurements on this topic have been published [38].

Dependent on the tertiary structure of proteins, denaturation does not necessarily increase the hydrodynamic radius. In cases where the native structure comprises stiff lobes such as in calmodulin, denaturation can actually lead to a more globular shape with smaller hydrodynamic radius (cf. Fig. 8).

It is also conceivable that the fluorescence properties of a particular substance are directly conformation dependent. Widengren and Schwille [39] applied FCS to characterize the photoinduced isomerization and backisomerization of the cyanine dye Cy5. As cyanine dyes are commonly used for a variety of different (fluorescence) applications, the photodynamics of this substance is of general interest.

Another member of the cyanine family, merocyanine 540, was more closely investigated by Widengren and Seidel [40]. This dye is used as therapy agent. It has been found to inactivate pathogenic viruses in blood and sensitize the selective photoinactivation of leukemia and lymphoma cells. In the photodynamic action of merocyanine 540, singlet oxygen generated during triplet quenching is believed to be of major importance. Therefore, the authors tried to characterize the competing reactions of isomerization and intersystem crossing to identify a way to favor the latter and thus optimize the photodynamic action.

Kim et al. [41] finally managed to apply FCS even to immobilized RNA three-helix junctions by combining the technique with fluorescence resonance energy transfer (FRET). Fluorescence fluctuations on the longest time scales are no longer due to the diffusion of molecules in and out of the focal volume. Transitions between the open and the closed conformation of these RNA helices caused FRET-based fluorescence fluctuations on millisecond time scales. Conventional FCS on freely diffusing molecules is not applicable in this case, in which the time scale of molecular transitions is larger than the diffusion time through the focus. By elegantly extending the scope of FCS, the conformational changes of these molecules initiating the folding of the $30 \mathrm{~S}$ 

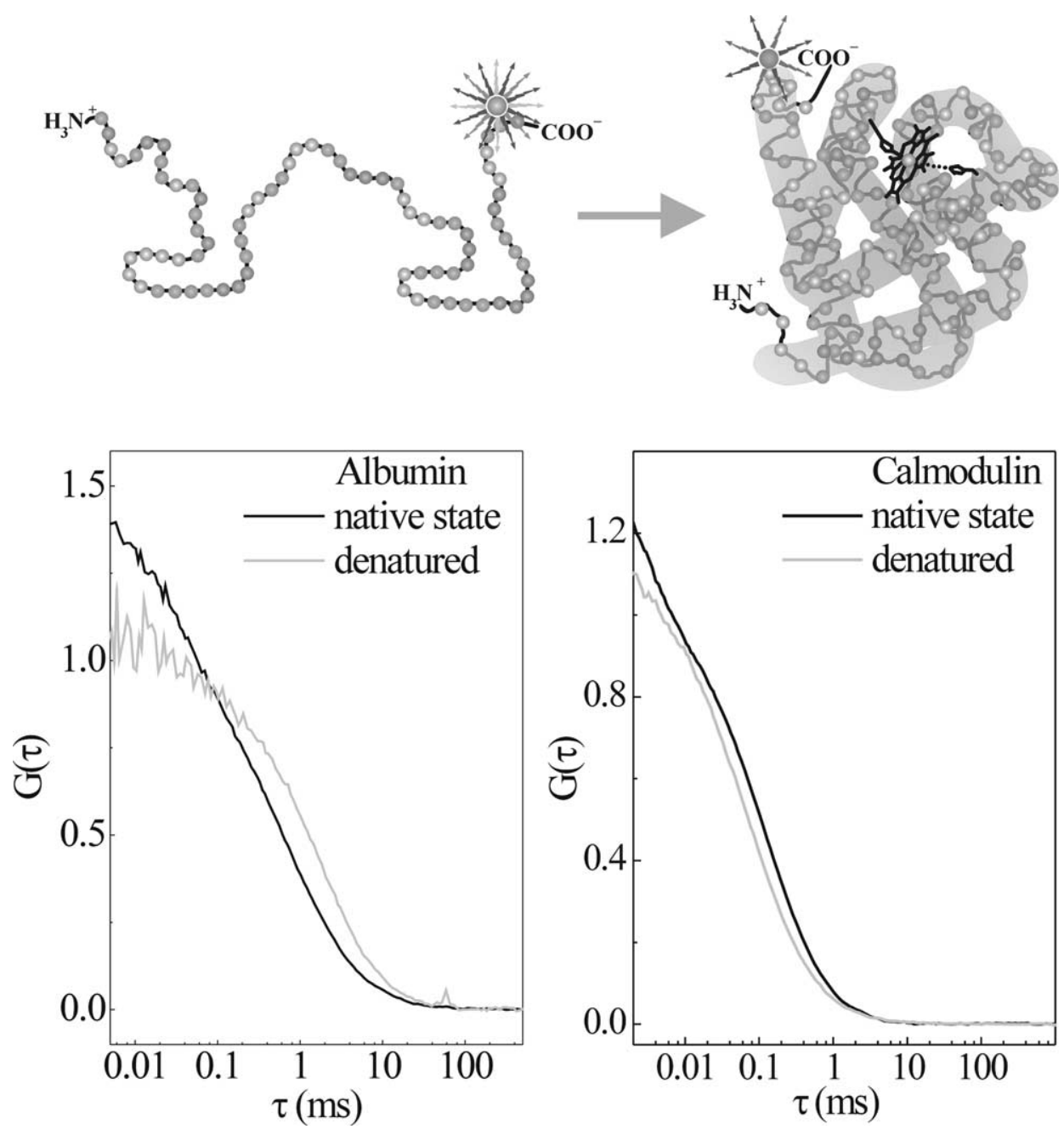

Fig. 8. $R_{\mathrm{h}}$ is usually smaller for globular molecules than for elongated ones (top) and can thus be used to determine the folding state of proteins. (Bottom) Examples for changes in diffusion coefficients following protein denaturation. Albumin in the denaturated state exhibits longer diffusion times $\tau_{\mathrm{d}}$, while denaturated calmodulin tends to diffuse faster with shorter $\tau_{\mathrm{d}}$.

ribosomal subunit were found to depend crucially on $\mathrm{Mg}^{2+}$ and $\mathrm{Na}^{+}$concentrations.

\subsection{Intramolecular dynamics probing the molecular microenvironment}

Fast fluctuations in the fluorescence emission need not be caused by isomerization reactions. There is a much more common photophysical phenomenon leading to blinking on fast time scales: At the high excitation rates applied for FCS measurements, the quantum mechanically forbidden transition between the first excited singlet and the triplet state becomes increasingly probable. This so-called intersystem crossing in fact occurs in most standard dyes, due to their complex photophysical nature. The excited-state lifetime for the triplet state is usually several orders of magnitude larger than the fluorescence lifetime. It can be up to several microseconds. Mathematically, the corresponding fast dynamics part of the autocorrelation function can be described by Eq. (5). The triplet state parameters depend mainly on the excitation intensity, but are also influenced by the chemical environment of the dye. Molecular oxygen is one of the most common triplet-state quenchers, and several heavy metal ions have been shown to alter the triplet-state kinetics [42]. Being dependent on intrinsic photophysical properties, these parameters are neither influenced by the molecular mobility nor affected by the size of the detection volume. Fig. 9 exemplifies this by showing two correlation curves of the dye tetramethylrhodamine, taken at the same power levels, but under different atmospheres, air and He. As oxygen preferentially quenches the triplet state, it can be seen that the relative fractions $\mathrm{T}$ of molecules occupying the triplet state and the triplet relaxation rates $\tau_{\mathrm{T}}$ are well distinguished in both cases. This strong sensitivity of certain fluorescent probes to oxygen or ion concentrations could potentially be useful for probing the intracellular environment. 


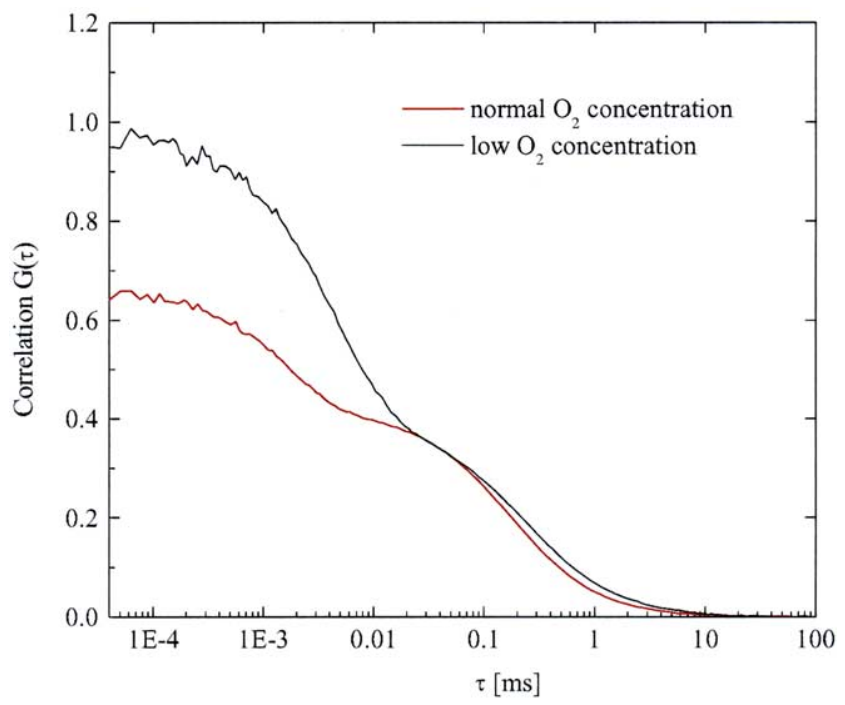

Fig. 9. The most prominent fast dynamics are photophysical transitions between the first excited singlet and the triplet states. Being quantum mechanically forbidden, these triplet excited states exhibit by far longer lifetimes than the respective singlet states, often of the order of microseconds. These parameters are neither influenced by the molecular mobility nor affected by the size of the detection volume, but a strong dependence on the illumination intensity and on certain environmental conditions can be observed. Here, two correlation curves of the dye tetramethylrhodamine are shown. They were taken at the same power levels, but under different atmospheres, air and He. As oxygen preferentially quenches the triplet state, it can be seen that the relative fractions $\mathrm{T}$ of molecules occupying the triplet state and the triplet relaxation rates $\tau_{\mathrm{T}}$ are well distinguished in both cases.

There is quite a substantial number of chromophores exhibiting $\mathrm{pH}$-dependent emission characteristics, which in conjunction with FCS measurements can be useful tools to probe the local $\mathrm{pH}$ on a singlemolecule level. One of the most popular of these potential single-molecule $\mathrm{pH}$ meters is the cloneable green fluorescent protein (GFP), particularly suited to intracellular applications. Predominantly the long-wavelength mutants EGFP (enhanced GFP) and yellow fluorescent protein (YFP) show interesting emission dynamics (so-called "flickering") on time scales between micro- and milliseconds, being easily accessible by FCS [28]. Haupts et al. [43] identified a reversible protonation of the chromophore of EGFP to be responsible for the flickering phenomenon, which is reflected by a pronounced shoulder on short time scales in the autocorrelation curves in Fig. 10. In bulk solutions of EGFP, the average fluorescence emission is known to decrease to zero at low $\mathrm{pH}\left(p \mathrm{~K}_{\mathrm{a}}=5.8\right)$ paralleled by a decrease of the absorption at $488 \mathrm{~nm}$ and an increase at $400 \mathrm{~nm}$. In contrast to these macroscopic effects, FCS thus reveals the microscopic nature of such an overall decrease by determining the emission pattern, i.e., the fast changes between dark and bright periods.
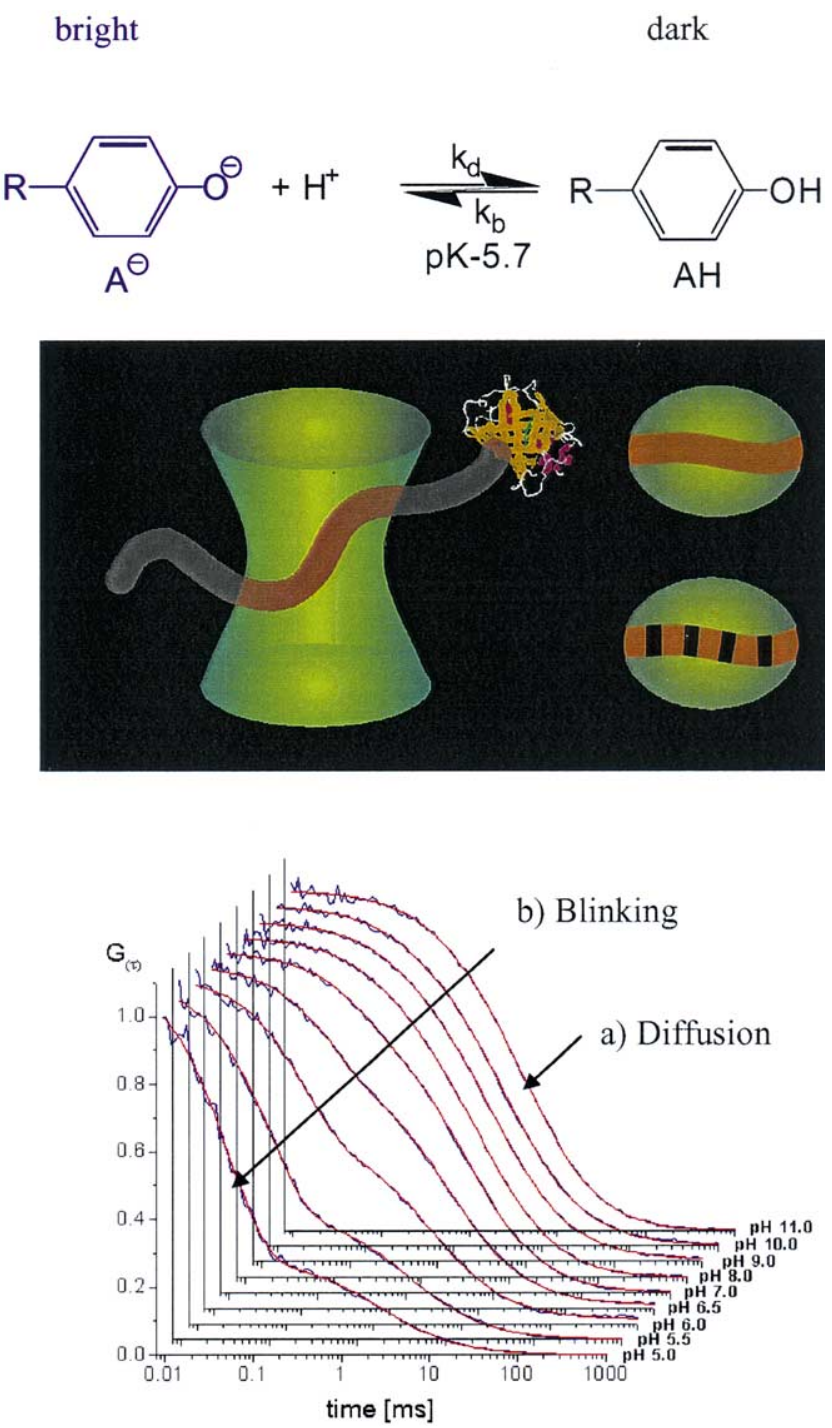

Fig. 10. (Top) Reaction scheme showing (middle) the transition from bright (deprotonated) to dark (protonated) state of the dye for excitation at $488 \mathrm{~nm}$. (Bottom) Autocorrelation curves measured in buffer solutions of different $\mathrm{pH}$. With decreasing $\mathrm{pH}$, the fast-blinking dynamics, which can be seen as first decay on fast time scales, becomes more and more pronounced.

The emission dynamics of the red fluorescent protein DsRed, isolated from Discosoma corals, has also been studied by FCS [44]. It was verified that this protein exhibits strong light-dependent flickering similar to the behavior of several yellow-shifted mutants of GFP. FCS data recorded at different intensities and excitation wavelengths suggest that this protein appears in at least three interconvertible states under equilibrated conditions. Light absorption induces transitions and/or cycling between these states on time scales of several tens to several hundreds of microseconds, dependent on excitation intensity. In analogy to GFP, this light-induced dynamic behavior suggests that population of the excited state is related to conformational rearrangements of the chromophore. 


\subsection{Investigating intermolecular reactions}

Recapitulating the various aspects of mobility analysis discussed above, it is evident that FCS is a versatile tool for all applications that somehow involve the determination of diffusion or transport coefficients. Provided that the changes in diffusion coefficients are large enough, association or dissociation reactions also may be followed. Assuming that a change in the diffusion coefficient of a factor of two would be sufficient to distinguish particles by FCS, one can estimate the necessary changes in the hydrodynamic radius via the Stokes-Einstein relationship. Approximating the particle by a homogeneous sphere, the hydrodynamic radius will scale with the cubic root of the molecular weight. Therefore, a globular protein would need to gain about eight times its own mass to follow its association process. Despite its attractiveness, this approach is thus applicable only to a limited number of possible reaction systems.

An ideal reaction system consists of one small, labeled ligand and a comparatively large, nonfluorescent counterpart, as depicted in Fig. 11. Analyzing the system is especially easy, if only one fluorescent species (e.g., fully free or fully bound ligand) is present at the start and the end of the association process. During the reaction, the percentage of the complex will continuously increase, until chemical equilibrium is reached or one species has completely reacted. Therefore, it is possible to determine the diffusion time of the labeled ligand from a reference measurement. Then, the other curves can be fitted with two diffusing components, fixing the diffusion time of the smaller component to the value determined beforehand [9]. Kinjo and Rigler [6] first established this principle to follow the binding kinetics of short fluorescent DNA probes to a longer DNA target; the same measurement scheme was further applied to a comparison of hybridization kinetics of DNA probes with different binding sites to folded RNA [8]. Mobility analysis has meanwhile proven to be an extremely powerful tool for a large variety of ligandreceptor systems [45-47].

Bismuto et al. [48] investigated the binding of ANS to native and partly folded tuna apomyoglobin by using yet another florescence property that changes upon binding. ANS, a charged hydrophobic naphtalene dye, is strongly fluorescent when bound to the protein and essentially dark in aqueous solution. The reaction rate has been determined as a function of concentrations of both ANS and apomyoglobin, temperature, and $\mathrm{pH}$.

Unfortunately, such intelligent probes are not available for all bimolecular reactions. The obvious solution to this dilemma is dual-color cross-correlation. Although the required setup is considerably more expensive, due to the second laser and detector, and more difficult to align, this technique is much more versatile,

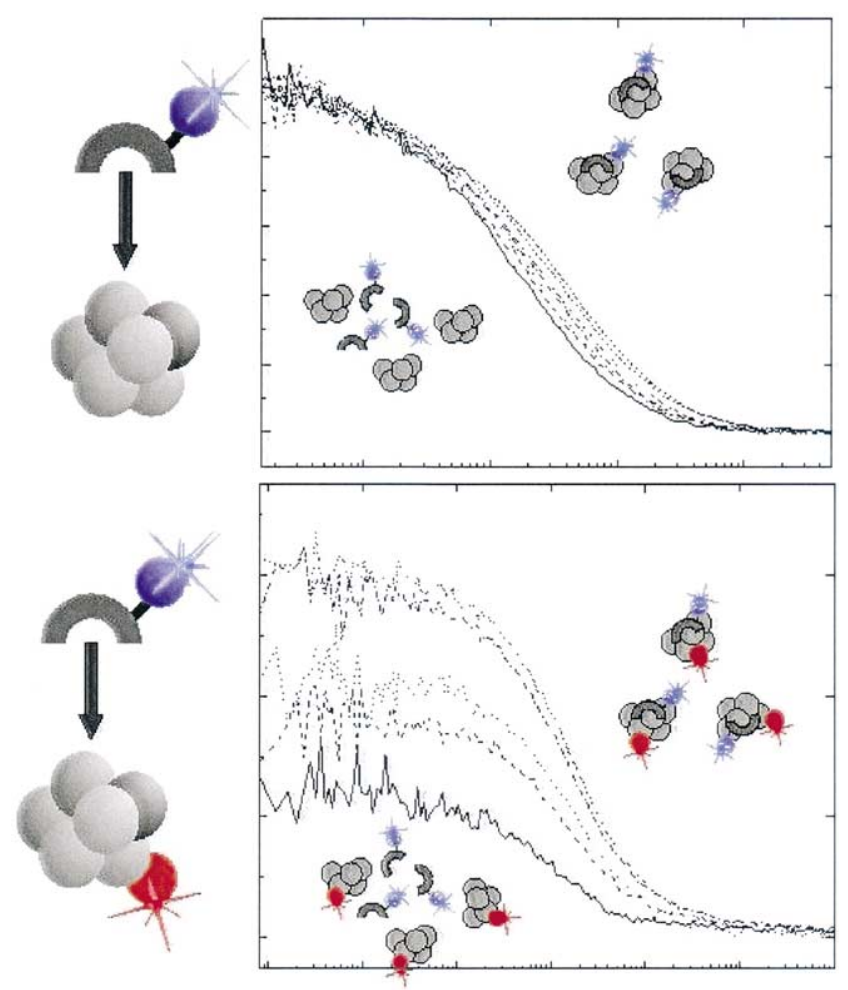

Fig. 11. Changes in diffusion time of a small fluorescently labeled ligand on binding to a heavy protein. It is evident that the more massive particles will move more slowly and thus exhibit a smaller diffusion coefficient and a longer average diffusion time. But as this change approximately scales inversely with the cubic root of the molecular mass, the gain in weight has to be approximately an order of magnitude before a significant change in diffusion time can be determined. In contrast to this, the changes in the dual-color cross-correlation observed upon binding are much more pronounced and easier to interpret.

and data analysis can be significantly simplified. The most important parameter is now simply the cross-correlation amplitude, which is a direct measure for the concentration of double-labeled particles diffusing through the focal volume. In principle, one simply focuses on the occurrence of coincident fluctuations in the two emission channels, induced by concerted motion of spectrally distinguishable labels. Under ideal conditions (i.e., no cross talk between the detectors), the amplitude $G_{x}(0)$ is zero unless double-labeled particles are present in the sample.

The tremendously enhanced detection specificity was first shown on association reactions of two small complementary DNA oligonucleotides carrying green and red labels [12]. It was demonstrated that the absolute concentration of the dimer could be directly monitored by cross-correlation.

On the basis of dual-color cross-correlation, Kettling et al. [15] demonstrated a biologically very attractive approach to characterize enzyme kinetics at extremely low enzyme concentrations $(>1.6 \mathrm{pM})$. The assay to be 
tested was the cleavage of double-labeled dsDNA by EcoRI restriction endonuclease.

As yes/no decisions about enzyme activity are possible just by recording the cross-correlation amplitude, this technique is extremely attractive for fast screening applications. The acquisition time per sample could be reduced to less than $1 \mathrm{~s}$ without substantially impairing the reliability [49]. Indeed, FCS has been predicted to have a very promising future as one of the standard techniques for high-throughput screening [50,51]. One other intriguing future application in the context of FCS is gene sequencing, which has often been discussed and serves as a starting point for other research. A DNA strand consisting completely of fluorescently labeled nucleotides is immobilized at one end in a laminar flow, e.g., by attachment to an optically trapped polystyrene bead. Then the DNA is sequentially shortened at the other end by an exonuclease, one nucleotide at a time. These are then detected and identified as the flow through two laser foci (spatial cross-correlation) [50,5254].

Despite the many exciting features of FCS, one has to point out that especially in custom-made setups, there are many different pitfalls for an unexperienced scientist, with regard to proper alignment and subsequent correct interpretation of the measured curves. Some of the problems, in particular for cross-correlation, are explained in more detail by Weidemann et al. [55].

The most elegant way of doing dual-color cross-correlation analysis is two-photon excitation. This was first accomplished in the Schwille laboratory [16] (cf. above). The extremely promising concept was experimentally demonstrated with the established EcoRI endonuclease assay originally introduced for one-photon FCCS, using rhodamine green and texas red.

\subsection{Measurements in living cells}

When measuring on a molecular scale, even such an apparently simple organism as a single cell is a highly complicated system. There are various possibilities for a molecule to be transferred from the place it is produced to where it is needed. Obviously, the simplest means of transportation is diffusion along a concentration gradient. Nevertheless, it is generally much more effective for the cell to use directed transport along an internal tubular network. Much work is presently devoted to characterize the endocytic and secretory trafficking pathways of proteins or vesicles or receptor internalization in signal transduction. FCS is well suited to distinguishing between diffusive and oriented motions of dilute molecules on a submillisecond time scale.

As an example, the mobility of GFP targeted to the plastid stroma in tubular structures interconnecting separate plastids in vascular plants was investigated using FCS [56]. As exchange of GFP between different interconnected plastids was obviously possible, the major task of FCS analysis consisted in revealing the nature of the extremely slow transport phenomenon. For this study, two-photon excitation was chosen because of the higher background suppression and increased IR light tolerance by plant cells. Positioning the laser focus directly on tubular structures, it could be shown that most of the GFP was contained within vesicular structures. Thus, it is indeed actively transported, although in rather large batches, and superimposed to anomalous diffusion. Especially when measuring in small cytosolic compartments, however, the correct model is crucial. Gennerich and Schild [57] proposed a mathematical description for spatially confined diffusion and verified the model by measuring within dendrites of cultured neurons.

Because of the complex nature of biological systems, evidence from various measurements is often required to clearly rule out any artifacts before the motility can be termed truly anomalous. The cytosolic curve in Fig. 7 is an example of anomalous diffusion caused by the interactions of the dye with the cellular environment. The increased viscosity of the cytosol compared to the buffer solution would mainly result in a parallel shift of the autocorrelation curve toward longer times. Here, however, the form of the curve is changing strongly, so that interactions with intracellular membranes must be suspected $[17,25]$. If the dye is replaced by the rather inert GFP, normal diffusion in the cytoplasm can be observed [32]. The diffusion coefficient is three to four times reduced relative to buffer measurements, which corresponds to the increased viscosity. As has been shown recently, the diffusion of EGFP in the nucleus suddenly becomes anomalous also, although it is perfectly homogeneous in the cytosol [18]. This may justify the assumption that the topology of cellular organelles has a nonnegligible effect on the diffusional properties. In Fig. 12, another set of typical FCS mobility measurements on cells is presented. As can be seen from the images taken with the laser scanning microscope, these human embryonic kidney (HEK) cells contained fluorophores in different compartments. The two-dimensional diffusion of lipid probe DiI in the plasma membrane is depicted on the left, whereas the three-dimensional diffusion of EGFP in the cytoplasm of the same cell line can be seen on the right. Since calibration measurements in aqueous solution yielded the diffusion time $\tau_{\mathrm{d}}$ of EGFP and thus also the corresponding diffusion coefficient of $8 \times 10^{-7} \mathrm{~cm}^{2} / \mathrm{s}$, the relative viscosity of the cytosol with respect to water can be easily determined. The measured diffusion times, $\tau_{\mathrm{d}}$, in the cytosol are about three to four times larger than those in water, indicating a three- to fourfold higher viscosity. This means that the diffusion coefficients are approx. $2.3 \times 10^{-7} \mathrm{~cm}^{2} / \mathrm{s}$. With regard to the behavior of the membrane probe DiI on living cells, its two-dimensional 

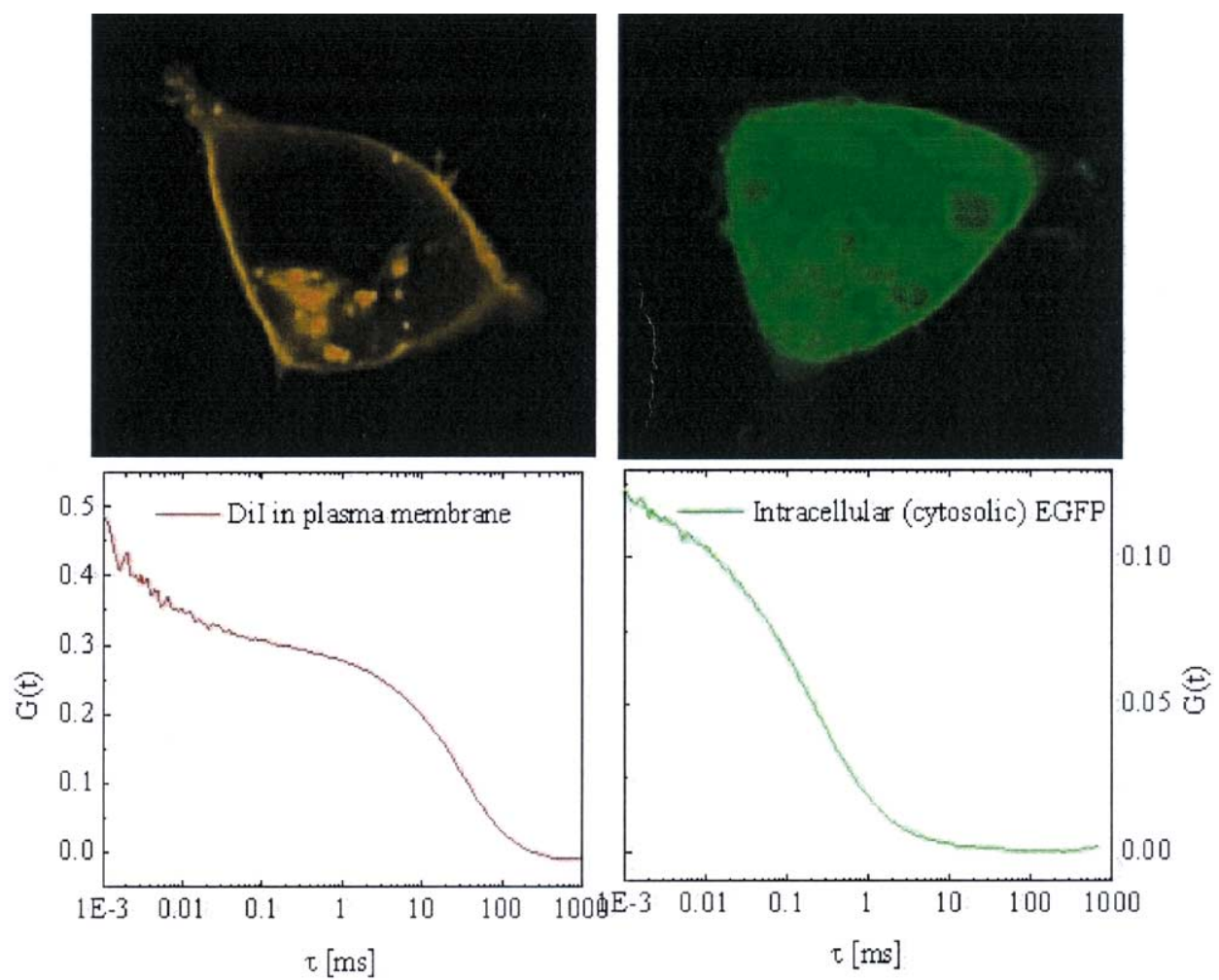

Fig. 12. Typical FCS mobility measurements performed on cells containing fluorophores in different cellular compartments. (Left) Two-dimensional diffusion of lipid probe DiI in the plasma membrane of human embryonic kidney cells. (Right) Three-dimensional diffusion of autofluorescent protein EGFP in the cytoplasm of the same cell line. Comparing the two autocorrelation curves, one cannot fail to notice that the diffusion in the plasma membrane is much slower. Indeed, two-dimensional diffusion of the membrane probe DiI on living cells can be described only as anomalous with $\alpha \approx 0.7$.

diffusion can be described only as anomalous with $\alpha \approx 0.7$.

Another situation where the mobility of molecules can be altered dramatically is when membrane binding is involved. When a molecule adheres to the membrane itself or to membrane proteins such as receptors, both the diffusion type (two-dimensional instead of three-dimensional) and the characteristical time scale are changed. Recently, the first applications in cell cultures were reported, investigating the binding and displacement of proinsulin C-peptide [58] and epidermal growth factor [59] to and from cell membranes. This work may be a first step toward the ultimate goal of large-scale drug screening in cell cultures.

As some substances are extremely scarce or only small fractions are functional, the enhanced sensitivity and spatial resolution of especially two-photon FCS may prove an extremely versatile tool for investigating processes in living cells. Two-photon excitation allows specific illumination of interesting sites in the living cell and eliminates photodamage effects in off-focus areas. By applying two-photon excitation to intracellular FCS [25], it could indeed be verified that, in comparison to conventional one-photon FCS, two-photon excitation at the same signal levels minimizes photobleaching in spatially restrictive cellular compartments, thereby preserving long-term signal acquisition. It is easily conceivable that future measurements in living cells and organisms will be routinely performed with single-molecule sensitivity [14].

\section{Concluding remarks}

It has been shown that FCS is indeed an extremely versatile yet noninvasive technique combining high sensitivity with statistical confidence. It is equally suited to both in vitro and in vivo systems. Due to the inherent sectioning, especially two-photon excitation can be considered ideal for intracellular measurements. As meanwhile the second generation of commercial setups has become available, initial experimental difficulties can be easily overcome. Perhaps this is the truest indication for the promising past and future development of the method and its gradual incorporation into the number of well-established standard techniques for the biosciences. Nevertheless, with the number of potential and actual users increasing continuously, the technique is still subject to further development and improvements. Looking at the achievements of the past 30 years, this leaves 
indeed much to be hoped for in future, maybe in combination with other promising fluorescence techniques.

\section{Acknowledgments}

The authors are grateful to Katrin Heinze for helpful discussion and the spectra depicted in Fig. 6. Financial support was provided by the Volkswagen Foundation (Grant No. I/76 676), the German Ministry of Education and Research (Grants No. 0311845 and No. 16SV1257), and Evotec OAI (Hamburg, Germany).

\section{References}

[1] D. Magde, E.L. Elson, W.W. Webb, Phys. Rev. Lett. 29 (1972) $705-708$.

[2] E.L. Elson, D. Magde, Biopolymers 13 (1974) 1-27.

[3] D. Magde, E.L. Elson, W.W. Webb, Biopolymers 13 (1974) $29-61$.

[4] R. Rigler, J. Widengren, Bioscience 3 (1990) 180-183.

[5] R. Rigler, U. Mets, J. Widengren, P. Kask, Eur. Biophys. J. 22 (1993) 169-175.

[6] M. Kinjo, R. Rigler, Nucleic Acids Res. 23 (1995) 1795-1799.

[7] B. Rauer, E. Neumann, J. Widengren, R. Rigler, Biophys. Chem. 58 (1996) 3-12.

[8] P. Schwille, F. Oehlenschläger, N.G. Walter, Biochemistry 35 (1996) 10182-10193.

[9] P. Schwille, J. Bieschke, F. Oehlenschläger, Biophys. Chem. 66 (1997) 211-228.

[10] J. Widengren, U. Mets, R. Rigler, J. Phys. Chem. 99 (1995) $13368-13379$.

[11] M. Eigen, R. Rigler, Proc. Natl. Acad. Sci. USA 91 (1994) 57405747.

[12] P. Schwille, F.J. Meyer-Almes, R. Rigler, Biophys. J. 72 (1997) 1878-1886.

[13] J. Bieschke et al., Proc. Natl. Acad. Sci. USA 97 (2000) 54685473.

[14] P. Schwille, U. Kettling, Curr. Opin. Biotech. 12 (2001) 382-386.

[15] U. Kettling, A. Koltermann, P. Schwille, M. Eigen, Proc. Natl. Acad. Sci. USA 95 (1998) 1416-1420.

[16] K.G. Heinze, A. Koltermann, P. Schwille, Proc. Natl. Acad. Sci. USA 97 (2000) 10377-10382.

[17] P. Schwille, J. Korlach, W.W. Webb, Cytometry 36 (1999) 176-182.

[18] M. Wachsmuth, W. Waldeck, J. Langowski, J. Mol. Biol. 298 (2000) 677-689.

[19] J. Widengren, R. Rigler, Bioimaging 4 (1996) 149-157.

[20] A.G. Palmer III, N.L. Thompson, Biophys. J. 51 (1987) 339-343.

[21] P. Schwille, K.G. Heinze, Chemphyschem 2 (2001) 269-272.

[22] P. Schwille, Cell Biochem. Biophys. 34 (2001) 383-408.

[23] W. Denk, J.H. Strickler, W.W. Webb, Science 248 (1990) 73-76.

[24] W. Denk, D.W. Piston, W.W. Webb, in: J.B. Pawley (Ed.), Handbook of Biological Confocal Microscopy, Plenum Press, New York, 1995, pp. 445-458.

[25] P. Schwille, U. Haupts, S. Maiti, W.W. Webb, Biophys. J. 77 (1999) 2251-2265.
[26] C. Xu, W.W. Webb, in: J.R. Lakowicz (Ed.), Topics in Fluorescence Spectroscopy, vol. 5, Plenum Press, New York, 1997, pp. 471-540.

[27] M. Lippitz, W. Erker, H. Decker, K.E. van Holde, T. Basche, Proc. Natl. Acad. Sci. USA 99 (2002) 2772-2777.

[28] P. Schwille, S. Kummer, A.A. Heikal, W.E. Moerner, W.W. Webb, Proc. Natl. Acad. Sci. USA 97 (2000) 151-156.

[29] N.L. Thompson, in: J.R. Lakowicz (Ed.), Topics in Fluorescence Spectroscopy, vol. 1, Plenum Press, New York, 1991, pp. 337378.

[30] M. Kinjo, G. Nishimura, Bioimaging 5 (1997) 134-138.

[31] N.O. Petersen, D.C. Johnson, M.J. Schlesinger, Biophys. J. 49 (1986) 817-820.

[32] P. Dittrich, F. Malvezzi-Campeggi, M. Jahnz, P. Schwille, Biol. Chem. 382 (2001) 491-494.

[33] T.J. Feder, I. Brustmascher, J.P. Slattery, B. Baird, W.W. Webb, Biophys. J. 70 (1996) 2767-2773.

[34] J.-P. Bouchaud, A. Georges, Phys. Rep. 195 (1990) 127-293.

[35] M.J. Saxton, Biophys. J. 64 (1993) 1766-1780.

[36] J. Korlach, P. Schwille, W.W. Webb, G.W. Feigenson, Proc. Natl. Acad. Sci. USA 96 (1999) 8461-8466.

[37] D. Magde, W.W. Webb, E.L. Elson, Biopolymers 17 (1978) 361376.

[38] M. Borsch et al., FEBS Lett. 437 (1998) 251-254.

[39] J. Widengren, P. Schwille, J. Phys. Chem. A 104 (2000) 64166428.

[40] J. Widengren, C.A.M. Seidel, Phys. Chem. Chem. Phys. 2 (2000) 3435-3441.

[41] H.D. Kim et al., Proc. Natl. Acad. Sci. USA 99 (2002) 4284-4289.

[42] J. Widengren, R. Rigler, Cell Mol. Biol. 44 (1998) 857-879.

[43] U. Haupts, S. Maiti, P. Schwille, W.W. Webb, Proc. Natl. Acad. Sci. USA 95 (1998) 13573-13578.

[44] F. Malvezzi-Campeggi, M. Jahnz, K.G. Heinze, P. Dittrich, P. Schwille, Biophys. J. 81 (2001) 1776-1785.

[45] J. Schüler, J. Frank, U. Trier, M. Schafer-Korting, W.A. Saenger, Biochemistry 38 (1999) 8402-8408.

[46] T. Wohland, K. Friedrich, R. Hovius, H. Vogel, Biochemistry 38 (1999) 8671-8681.

[47] E. Margeat et al., J. Mol. Biol. 306 (2001) 433-442.

[48] E. Bismuto, E. Gratton, D.C. Lamb, Biophys. J. 81 (2001) 35103521.

[49] A. Koltermann, U. Kettling, J. Bieschke, T. Winkler, M. Eigen, Proc. Natl. Acad. Sci. USA 95 (1998) 1421-1426.

[50] S. Maiti, U. Haupts, W.W. Webb, Proc. Natl. Acad. Sci. USA 94 (1997) 11753-11757.

[51] L. Silverman, R. Campbell, J.R. Broach, Curr. Opin. Chem. Biol. 2 (1998) 397-403.

[52] K. Dörre et al., Bioimaging 5 (1997) 139-152.

[53] K. Dörre et al., J. Biotechnol. 86 (2001) 225-236.

[54] K. Dörre, J. Stephan, M. Eigen, Single Molecules 2 (2001) 165 175.

[55] T. Weidemann, M. Wachsmuth, M. Tewes, K. Rippe, J. Langowski, Single Molecules 3 (2002) 49-61.

[56] R.H. Köhler, P. Schwille, W.W. Webb, M.R. Hanson, J. Cell Sci. 113 (2000) 3921-3930.

[57] A. Gennerich, D. Schild, Biophys. J. 79 (2000) 3294-3306.

[58] R. Rigler et al., Proc. Natl. Acad. Sci. USA 96 (1999) 13318 13323.

[59] A. Pramanik, R. Rigler, Biol. Chem. 382 (2001) 371-378. 